\title{
Estresse ocupacional: estudo multifuncional com profissionais da saúde de um hospital de Araçatuba (SP)
}

\author{
Occupational stress: multifunctional study in health care professionals \\ of a hospital in Araçatuba (SP) \\ Estrés ocupacional: estudio multifuncional con profesionales de la salud \\ de um hospital de Araçatuba (SP) \\ Diogo REATTO \\ Daniela Cristina Celestino de Almeida ARAújo
}

\author{
${ }^{I}$ Doutorando em Administração - Área de Gestão Humana e Social nas Organizaçõe, Universidade Presbiteriana Mackenzie, São Paulo-SP, Brasil \\ Mestre em Administração - Gestão Humana e Social; Especialista em Gestão de Pessoas no Setor Público; \\ Graduação em Administração e Comércio Exterior \\ Professor dos Cursos de Administração e Tecnologia em Gestão de Recursos Humanos, \\ Centro Universitário Toledo - UNITOLEDO, Araçatuba-SP, Brasil \\ ${ }^{2}$ Especialista em Gestão Empresarial pelo Centro Universitário Toledo - UNITOLEDO, Araçatuba-SP, Brasil \\ Tecnóloga em Gestão de Recursos Humanos pelo Centro Universitário Toledo - UNITOLEDO, Araçatuba-SP, Brasil
}

\begin{abstract}
Resumo
Introdução: A área da saúde e o ambiente hospitalar sofrem com a presença de fatores que marcam a realidade de qualquer outra organização, estando igualmente predispostos a ter profissionais acometidos de estresse ocupacional. A instabilidade, a insegurança no trabalho, a condições precárias de trabalho, as longas jornadas, os conflitos nas relações interpessoais com colegas, a alta demanda de serviços pela população, a crise do sistema de saúde em muitas localidades, entre outros, são fatores que alertam para a necessidade de um olhar cuidadoso para a saúde mental desses trabalhadores, cujo objetivo maior é o cuidado com o outro. Objetivo: Identificar o nível de estresse ocupacional que caracterize ou não a Síndrome de Burnout em profissionais da área de saúde de um hospital do município de Araçatuba (SP). Material e método: Aplicou-se o Maslach Burnout Inventory - Human Service Survey/MBI-HSS - a 39 profissionais de saúde de um hospital no município de Araçatuba/SP, entre eles 10 auxiliares de enfermagem, 17 técnicos de enfermagem, 5 auxiliares de farmácia, 5 enfermeiros e 2 médicos. Resultados: Identificou-se alto nível de burnout nas dimensões Exaustão Emocional e Despersonalização em auxiliares de Enfermagem, técnicos de Enfermagem, auxiliares de Farmácia e enfermeiros. A Realização Profissional desses profissionais alcançou nível médio. Entre os médicos, não se identificou prevalência de burnout. Conclusão: Os resultados confirmam o histórico de pesquisas brasileiras sobre o assunto, principalmente entre enfermeiros. Há necessidade de o setor de saúde rever suas políticas e práticas de gestão, incluindo programas de qualidade de vida no trabalho e educação continuada.
\end{abstract}

Descritores: Qualidade de Vida; Estresse Psicológico; Esgotamento Profissional.

\begin{abstract}
Introduction: The health field and the hospital environment suffer from the presence of factors that highlight the reality of any other organization, and both are predisposed to have stressed out professionals. Job instability, lack of job security condition, substandard job environment, conflict with colleagues, high demand of health care services by population, health sector crisis in many locations, and others, are factors that can draw a careful attention to the mental health of those professionals, whose main goal is to care for the other. Objective: This study aimed to identify the occupational stress level that features or not burnout syndrome in health care professionals of a hospital in Araçatuba/SP. Material and method: It was applied the instrument Maslach Burnout Inventory - Human Service Survey/MBI-HSS to 39 health care professionals, being 10 nursery auxiliaries, 17 nursery technicians, 5 pharmaceutical auxiliaries, 5 nurses and 2 doctors. Results: It was identified a high level of burnout syndrome in emotional exhaustion and depersonalization dimensions among nursery auxiliaries, nursery technicians, pharmaceutical auxiliaries and nurses. Personal accomplishment reached middle level. It was not identified burnout syndrome prevalence in the doctors studied. Conclusion: The results confirm the historical of Brazilian researches about occupational stress and burnout syndrome in health care professionals. There is urgency for the health sector to review its policies and management practices, including quality of life programs at work and continuing education.
\end{abstract}

Descriptors: Quality of life; Stress-Psychological; Burnout-Professional.

\section{Resumen}

Introducción: El área de la salud y el ambiente hospitalario sufren con la presencia de factores que marcan la realidad de cualquier otra organización, estando igualmente predispuestos a tener profesionales acometidos de estrés laboral. La inestabilidad, inseguridad en el trabajo, precariedad de las condiciones de trabajo, largas jornadas, conflictos en las relaciones interpersonales con colegas, alta demanda de servicios por la población en general, crisis del sistema de salud en muchas localidades, entre otros, son factores que alertan para la necesidad de una mirada más cuidadosa para la salud mental de estos trabajadores, cuyo objetivo mayor es el cuidado con el otro. Objetivo: Identificar el nivel de estrés laboral que caracterice o no el Síndrome de burnout en profesionales de la salud de un hospital de Araçatuba/SP. Material y método: Se aplicó el Maslach Burnout Inventory - Human Service Survey/MBI-HSS a 39 profesionales de salud de un hospital en el municipio de Araçatuba/SP, siendo 10 auxiliares de enfermería, 17 técnicos de enfermería, 5 auxiliares de farmacia, 5 enfermeros y 2 médicos. Resultados: Se identificó alto nivel de burnout en las dimensiones agotamiento emocional y despersonalización en auxiliares y técnicos de enfermería, auxiliares de farmacia y enfermeros. La realización profesional de estos profesionales alcanzó nivel mediano. Entre los médicos no se identificó burnout. Conclusión: Los resultados confirman el historial de investigaciones brasileñas sobre el asunto, principalmente entre enfermeros. El sector de salud debe rever sus políticas y prácticas de gestión, incluyendo programas de calidad de vida en el trabajo y educación continuada.

Descriptores: Calidad de Vida; Estrés Psicológico; Agotamiento Profesional. 


\section{INTRODUÇÃO}

Com o desenvolvimento contínuo das tecnologias e com a globalização, as organizações estão preocupadas em produzir com qualidade devido à constante exigência, competitividade e seletividade, desta forma, quem não atende às exigências de produção e oferta está propenso a ficar à margem do mercado. A qualidade dos produtos e serviços é influenciada pela capacitação, treinamento e motivação dos funcionários. As exigências do mercado e a competitividade levaram as organizações de todos os ramos de atividades, desde a industrial até aquelas que perpassam a educação e a saúde, a exigirem mais dos trabalhadores, e essa pressão trouxe consequências como o aumento dos acidentes de trabalho e afastamento por motivo de saúde como estresse, depressão e outras patologias.

Para afrontar estas circunstâncias delicadas que atingem o cenário laboral, desde 1950, cientistas sociais, líderes sindicais, empresários e governantes discutem e fazem avançar os estudos sobre a qualidade de vida no trabalho na busca de um conjunto de ações que permitam organizar o trabalho a fim de minimizar os efeitos negativos do emprego na saúde e no bem-estar geral dos trabalhadores ${ }^{1,2}$.

Quando nos preocupamos em discutir as patologias da ocupação, uma das mais proeminentes é o estresse. Compreende-se por estresse ocupacional um conjunto de perturbações ou instabilidade psíquica e orgânica provocado por diversos estímulos que vão desde a condição climática até as emoções e condições de trabalho. Na base da compreensão do conceito de estresse está o desequilíbrio, no caso, na relação entre trabalhador e ocupação. Entende-se, então, estresse ocupacional como o quadro de respostas pouco adequadas à estimulação física e emocional decorrente das exigências do ambiente de trabalho, das capacidades exigidas para realizá-lo e das condições do trabalhador ${ }^{3}$.

Os estudos sobre o estresse ocupacional permitem um melhor entendimento sobre as suas causas e contribuem para elucidar assuntos relacionados à saúde mental de diversa gama de ocupações. Particularmente, as profissões da área da saúde envolvem intensa atenção à pessoa que necessita cuidados ou àqueles que são dependentes, condição ideal para que esses profissionais experienciem altos níveis de estresse ${ }^{4}$.

Em alguns casos, o estresse ocupacional não tratado pode gerar a Síndrome de burnout caracterizada pelo esgotamento físico e psíquico em decorrência do trabalho ${ }^{3}$. A Síndrome de Burnout (SB) é um tipo de estresse ocupacional que acomete profissionais envolvidos com qualquer tipo de cuidado em uma relação de atenção direta, contínua e altamente emocional $^{5}$. Esta síndrome é definida como uma reação à tensão emocional crônica gerada a partir do contato direto e excessivo com outros seres humanos, particularmente quando estes estão preocupados ou com problemas ${ }^{6}$. O trabalhador se envolve afetivamente com seus clientes, desgasta-se e, num extremo, desiste e ou não suporta mais. A síndrome é entendida num conceito multidimensional que envolve três componentes: 1) a exaustão emocional, em que trabalhadores entendem que não podem dar mais de si mesmos e percebem o esgotamento da energia e dos recursos emocionais próprios devido ao contato diário com os problemas; 2) a despersonalização, a qual caracteriza-se por sentimentos e atitudes negativas e de cinismo com as pessoas destinatárias do trabalho, endurecimento afetivo e coisificação da relação; 3) a falta de envolvimento pessoal no trabalho, que é a tendência de uma evolução negativa no trabalho, afetando a habilidade para a sua realização.

Notou-se que os estudos que utilizam o modelo de Maslach no Brasil conferem certa confusão terminológica sobre o título das dimensões exaustão emocional, despersonalização e, em especial, falta de envolvimento pessoal. Por ser mais atual ${ }^{7,8}$, adotou-se, neste estudo, a seguinte nomenclatura para as subescalas: Exaustão Emocional (EE), Despersonalização (DP) e Realização Profissional (RP).

A subescala de exaustão emocional avalia as queixas sobre sentir-se no limite e exausto pelo trabalho. A subescala de despersonalização mede respostas impessoais e falta de empatia durante a atividade profissional. Já a subescala realização profissional identifica os sentimentos de competência e conquista de sucesso no trabalho?.

Verifica-se uma tendência das organizações hospitalares no investimento em estrutura física, mais especificamente na estética de suas instalações, com o intuito de gerar avaliação positiva por parte do usuário, estando essa questão relacionada ao mercado consumidor. No entanto, os profissionais que trabalham na instituição precisam, acima de tudo, de condições e organização de trabalho adequados, com suporte de seus supervisores, benefícios e políticas organizacionais que contemplem sua qualidade de vida $^{7}$. Assim, a SB em profissionais que atuam na área hospitalar é uma realidade preocupante, pois na ocorrência desta forma de adoecimento profissional, haverá comprometimento na qualidade da assistência que é prestada ao usuário e a toda a rede social envolvida ${ }^{7}$.

A natureza do trabalho, os vários vínculos empregatícios, a longa jornada de trabalho, a falta de autonomia e autoridade na tomada de decisões, a remuneração baixa em relação à responsabilidade, a 
complexidade das tarefas executadas, os recursos inadequados, as relações interpessoais e atendimento ao paciente ${ }^{10}$ contribuem na construção de um cenário profícuo para o desenvolvimento da SB em profissionais da saúde, independente de atuarem em áreas abertas ou fechadas da área hospitalar.

Apesar de a importância do assunto ser evidenciada pelos mais de cinco mil trabalhos levantados na base de dados PubMed, avaliar a presença de estresse ocupacional no ambiente de trabalho não é tarefa fácil. Devido à multiplicidade de conceitos $^{11}$ e metodologias de estudo ${ }^{12}$ para o estresse ocupacional, este trabalho adota o modelo de Maslach como norteador. Portanto, o objetivo desta pesquisa é identificar a prevalência da SB em profissionais da área da saúde de um hospital do município de Araçatuba (SP) utilizando uma amostra multifuncional, o que representa uma contribuição para os estudos devido ao inúmero inferior de trabalhos que se preocupam em comparar a prevalência da SB em diversas profissões ${ }^{7}$.

Para a consecução do objetivo geral, aplicou-se o Maslach Burnout Inventory - Human Service Survey - MBI-HSS - a 39 indivíduos ou profissinais da saúde, sendo eles, auxiliares de Enfermagem, técnicos de Enfermagem, auxiliares de Farmácia, enfermeiros e médicos. Todos os participantes foram previamente esclarecidos sobre os objetivos do estudo e assinaram o Termo de Consentimento Livre e Esclarecido TCLE.

\section{MATERIAL E MÉTODO}

Esta pesquisa epidemiológica tem caráter descritivo e quantitativo ${ }^{13}$, de modelo transversal. Como instrumento de pesquisa, utilizaram-se dois questionários. O primeiro questionário era composto por questões sócio-demográficas e serviu para a caracterização da amostra. O segundo questionário é o instrumento Maslach Burnout Inventory - Human Service Survey - MBI-HSS. A versão HSS do MBI foi traduzida e adaptada ao português por BenevidesPereira $^{14}$ e tem se mostrado a mais adequada para aplicação a profissionais da saúde em estudos que contemplem uma única função ou que sejam multifuncionais ${ }^{7}$.

Quanto à sua composição, o MBI-HSS é autoaplicado e compreende 22 afirmações que incidem sobre sentimentos e atitudes relacionados com o trabalho e com os clientes, divididos por três dimensões, apresentados no Quadro 1: Exaustão emocional (nove itens - 2, 5, 7, 8, 9, 10, 14, 16, 17), Despersonalização (cinco itens - 3, 13, 19, 20 e 21) e Realização Profissional (oito itens - 1, 4, 6, 11, 12, 15, 18 e 22). A resposta é dada sobre a frequência com que cada sentimento ocorre numa escala tipo Likert que varia entre 1 (discordo totalmente) a 7 (concordo totalmente).

Quadro 1. Descrição das dimensões da Síndrome de Burnout e seus respectivos itens

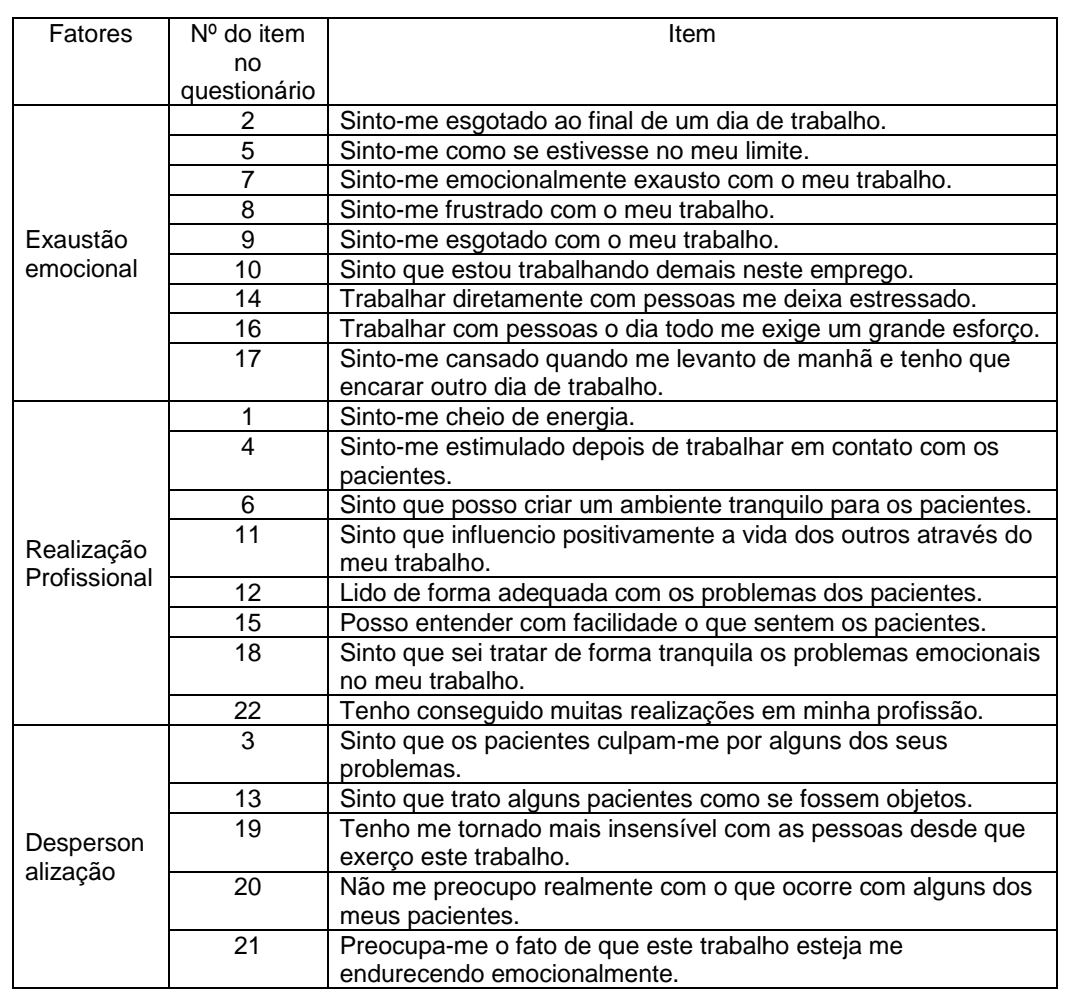

A Síndrome de Burnout é medida em níveis considerados baixos, médios e altos e não como presença ou ausência de sintomas ${ }^{15}$. Cada um dos fatores é analisado separadamente ${ }^{16}$ e seu valor é conseguido somando-se a pontuação da escala tipo Likert para cada um dos fatores, lembrando que o item 20, da dimensão Despersonalização, tem sua pontuação invertida antes da somatória final, pois a afirmação é semanticamente negativa.

Pela combinação do nível de cada um dos três fatores é que se obtém o nível de burnout do indivíduo (Tabela 1), o qual pode ser considerado preocupante ao encontrar-se nível médio em qualquer um dos fatores da escala ${ }^{17}$. Contudo, considera-se que um sujeito tem burnout quando obtém resultados elevados na dimensão exaustão emocional e despersonalização e baixa realização profissional ${ }^{18}$.

Baseado em Trigo ${ }^{9}$ (2010), adotou-se as duas formas existentes de se avaliar a prevalência da SB. A primeira consiste em atribuir os pontos de corte baixo, médio e alto de acordo com a pontuação somada das respostas da escala tipo Likert (Tabela 1).

Tabela 1. Parâmetros para os níveis de Burnout

\begin{tabular}{l|c|c|c}
\hline \multicolumn{1}{c|}{ Fatores } & Baixo & Médio & Alto \\
\hline Despersonalização & $1|-| 7$ & $7-\mid 13$ & $\uparrow 14$ \\
\hline Exaustão Emocional & $1 \mid-17$ & $17|-| 27$ & $\uparrow 27$ \\
\hline Realização Profissional & $\downarrow 32$ & $32|-| 39$ & $\uparrow 39$ \\
\hline
\end{tabular}

Fonte: $\operatorname{Noro}^{17}(2004$, p. 26)

A pontuação em cada subescala é considerada separadamente e suas pontuações não são somadas, logo, cada subescala é avaliada separadamente. A segunda opção de avaliação é a associação entre as três subscalas, gerando uma única variável 
categorizada em baixo, médio e alto quando as três subescalas apresentam, ao mesmo tempo, pontuações nesses intervalos, respectivamente. A exceção é para a intensidade alta da SB, quando duas subescalas recebem pontuação alta.

\section{RESULTADOS}

\section{- Caracterização da amostra}

Participaram do estudo 39 funcionários de um hospital do município de Araçatuba/SP, dentre eles 10 auxiliares de enfermagem, 17 técnicos de enfermagem, 5 auxiliares de farmácia, 5 enfermeiros e 2 médicos.

A média de idade é de 34,8 anos. Quanto à renda, 72,1\% ganham menos de $\mathrm{R} \$ 1900,00$ mensais. Quanto à experiência profissional, 43,6\% (17) já têm trabalham na área da saúde há mais de 10 anos.

A jornada de trabalho é em média de 49,6 horas/semana. Dos respondentes, 10 afirmaram ter dois empregos. Quatro deles são técnicos de enfermagem, os quais afirmaram ter 3 empregos, ou seja, trabalham os três períodos do dia, com uma carga horária de trabalho média semanal de 89 horas, ou seja, 12,7 horas por dia em média. Contudo, um dos médicos respondentes tem somente 1 vínculo empregatício com jornada semanal de 90 horas.

\section{- Índices médios de Burnout levantados}

No geral, as médias de Burnout obtidas para os profissionais de saúde pesquisados apresentaram os seguintes resultados de acordo com os parâmetros citados na Tabela 2: a subescala Despersonalização obteve um nível alto de 17,0; já a Exaustão emocional resultou em um nível alto de 30,5 e Realização Profissional apresentou uma média de 37,2, indicando um nível médio (Tabela 2).

Tabela 2. Índices médios de Burnout

\begin{tabular}{l|c|c}
\multicolumn{1}{c|}{ Fatores } & \multicolumn{2}{c}{ Níveis de Burnout } \\
\hline Despersonalização & Alto & 17,0 \\
\hline Exaustão Emocional & Alto & 30,5 \\
\hline Realização Profissional & Médio & 37,2 \\
\hline
\end{tabular}

Este estudo encontrou diferenças nas médias de SB entre as profissões. As maiores disparidades estão presentes nas três subescalas na comparação entre médicos com os demais profissionais investigados (Tabela 3).

Entre as demais categorias de profissões:

(a) há menor nível de exaustão emocional do técnico em Enfermagem, embora ainda considerado alto, em relação aos outros;

(b) mesmo considerado alto, o auxiliar de Farmácia tem o menor nível de despersonalização em comparação com os demais.

\begin{tabular}{l|l|c|c}
\multicolumn{4}{c}{ Tabela 3. Índices Médios de Burnout } \\
\multicolumn{4}{c}{ nos Cargos Estratégicos e Operacionais } \\
\hline \multicolumn{1}{c}{ Função } & \multicolumn{1}{c}{ Fatores } & Nível & Burnout \\
\hline \multirow{2}{*}{$\begin{array}{l}\text { Auxiliar de } \\
\text { Enfermagem }\end{array}$} & Despersonalização & Alto & 17,3 \\
\cline { 2 - 4 } & Exaustão Emocional & Alto & 33,5 \\
\cline { 2 - 4 } & Realização Profissional & Médio & 37,0 \\
\hline \multirow{2}{*}{$\begin{array}{l}\text { Técnico de } \\
\text { Enfermagem }\end{array}$} & Despersonalização & Alto & 16,6 \\
\cline { 2 - 4 } & Exaustão Emocional & Alto & 27,8 \\
\cline { 2 - 4 } & Realização Profissional & Médio & 36,3 \\
\hline \multirow{2}{*}{$\begin{array}{c}\text { Auxiliar de } \\
\text { Farmácia }\end{array}$} & Despersonalização & Alto & 15,0 \\
\cline { 2 - 4 } & Exaustão Emocional & Alto & 33,4 \\
\cline { 2 - 4 } & Realização Profissional & Médio & 37,2 \\
\hline \multirow{3}{*}{ Enfermeiro } & Despersonalização & Alto & 17,6 \\
\cline { 2 - 4 } & Exaustão Emocional & Alto & 33,2 \\
\cline { 2 - 4 } & Realização Profissional & Médio & 37,2 \\
\hline \multirow{2}{*}{ Médico } & Despersonalização & Alto & 22,5 \\
\cline { 2 - 4 } & Exaustão Emocional & Médio & 24,5 \\
\cline { 2 - 4 } & Realização Profissional & Alto & 46,0 \\
\hline
\end{tabular}

Ao se associar as três subescalas para verificar a prevalência de SB, pode-se considerar que há alto nível de burnout entre os auxiliares de Enfermagem, técnicos de Enfermagem, auxiliares de Farmácia e enfermeiros. Quanto aos médicos, a melhor opção de análise seria a isolada por dimensão, ou seja, estudar cada subescala separadamente, já que a pesquisa revelou alto nível de despersonalização, médio nível de exaustão profissional e alto nível de realização profissional. Embora haja dois níveis altos como resultado, a subescala Realização Profissional tem valor semântico invertido ao das demais categorias.

A próxima seção discute esses resultados com base na literatura encontrada.

\section{DISCUSSÃO}

Considerando-se as questões propostas pelo presente estudo, constatou-se que os níveis de Burnout apresentaram-se alto para a Despersonalização, alto para a Exaustão Emocional e baixo para a Realização Profissional em todas as categorias profissionais, com exceção à médica. Diante disso, a despersonalização é uma resposta de incompetência e/ou desesperança, a qual não se consegue mais administrar e, assim, o indivíduo passa a tratar as pessoas como objetos; esta fase viria como um sentimento de defesa contra a exaustão emocional, ou seja, seria uma tentativa dos trabalhadores em restabelecer a sua segurança no trabalho, o que na verdade não ocorre. E o menor nível de envolvimento com o trabalho é também um enfretamento defensivo, no qual ocorre uma mudança de atitudes e condutas. Geralmente, o menor nível de envolvimento com o trabalho aparece quando o profissional percebe uma diminuição da qualidade do seu trabalho e a sua realização profissional está comprometida, atacando justamente aqueles profissionais mais preocupados com a competência e por isso, mais exigentes ${ }^{17}$.

Pressupõe-se que o resultado obtido neste estudo confirma o histórico de pesquisas brasileiras sobre o assunto, principalmente entre enfermeiros. A primeira questão relevante a ser discutida é a relação de gênero entre os profissionais. A Enfermagem, em 
particular, é uma categoria essencialmente feminina ${ }^{11}$, o que deve ser considerado, pois as mulheres apresentam condições organizacionais mais negativas que os homens devido à falta de oportunidades de crescimento no ambiente de trabalho ${ }^{11}$. Ainda, a Enfermagem foi identificada como a quarta profissão mais estressante do mundo de acordo com a Health Education Authority ${ }^{19}$.

Outra questão é a própria natureza do trabalho dos profissionais da área da saúde, novamente destacando-se a área de Enfermagem. Os sentimentos de religiosidade presentes na profissão de enfermeiro podem influenciar os níveis de exaustão emocional à medida que fatores organizacionais como a falta de autonomia, más condições de trabalho, alta demanda de pacientes, longas jornadas de trabalho, baixa perspectivas de crescimento profissional, salários defasados e a dificuldade de delimitação entre o trabalho do enfermeiro e dos demais (técnicos, auxiliares, médicos) contrastam com a cobrança de uma imagem de profissional obediente, dócil, humilde, que respeita a hierarquia, disciplinado, com espírito servil e alienado ${ }^{19}$. Esses sentimentos de raiz religiosa podem contribuir para o aumento da percepção de realização profissional, quando esta está ligada a um sentimento moral de dever cumprido.

Ainda, chama-se a atenção para o fato de que os fatores organizacionais influenciam a SB quando o profissional se depara com frustrações ou sobrecarga de trabalho e aumenta seus esforços para cumprir esses desafios, compensando o sofrimento psicológico com esse esforço extra ${ }^{8}$. Estudo de Fernandes et al. ${ }^{20}$ (2008) com 15 enfermeiras concluiu que realmente estes profissionais tem desenvolvido estresse ocupacional decorrente do processo de trabalho hospitalar, marcado por condições precárias de trabalho e pelo aumento da jornada de trabalho.

Aos fatores organizacionais já levantados somam-se: os usuários necessitam cada vez mais tempo para serem atendidos, recursos econômicos escassos, legislação que não favorece o acesso à saúde para todos, limitado sistema de valorização e promoção de pessoal, medicalização de toda experiência humana e falta de profissionais ${ }^{8}$.

A maior parte dos estudos que tem o mesmo objetivo e objeto de estudos desta pesquisa encontrou níveis mais baixos de Realização Profissional. Justificativas para essa diferença poderiam estar em fatores organizacionais já identificados, porém que não são objeto de discussão neste trabalho, como oportunidades de crescimento, segurança no trabalho, salários, tipo de organização se pública ou privada.

Os estudos iniciais do burnout centraram-se nos profissionais de ajuda $^{21}$. De um modo geral, considera-se que todas as funções que envolvem complexas interações com outros indivíduos, sejam eles clientes, colegas ou pacientes, são caracterizadas por um conjunto de exigências interpessoais, às quais, a exposição crônica dos trabalhadores desencadeia a SB.

Na continuação da discussão sobre os maiores níveis de Realização Profissional encontrado nesta pesquisa, as dimensões de exaustão e despersonalização formam o núcleo central do burnout $^{22}$. Com efeito, diversos autores têm sugerido que a realização profissional reflete uma característica da personalidade, em vez de constituir um componente específico do burnout. Empiricamente, esta perspectiva é sustentada pelas baixas correlações da realização profissional com as outras duas dimensões, e pelo fato de a despersonalização parecer desenvolver-se em resposta à exaustão, enquanto a realização profissional parece desenvolver-se de modo independente e em paralelo.

Os fatores organizacionais voltam à discussão para justificar a Exaustão Emocional em auxiliares de Enfermagem e de Farmácia. Da mesma forma que estudo de Schmidt et al. ${ }^{11}$ (2009) afirma que os enfermeiros são os responsáveis pela administração e gestão de pessoal, pelo gerenciamento da assistência de enfermagem, pelo gerenciamento de conflitos e insatisfações e ainda que estão em nível superior na hierarquia institucional em relação aos demais trabalhadores da Enfermagem, problemas justamente nessa relação delicada e intrincada de trabalho podem estar sobrecarregando os demais funcionários, os quais estariam se sentindo sobrecarregados por ações inapropriadas, como alta descentralização por parte de suas chefias imediatas. Gomes, Cabanelas ${ }^{23}$ (2009) também encontraram nos fatores organizacionais as evidências para exaustão emocional em enfermeiras, nos enfermeiros mais novos, nos trabalhadores com contratos a prazo, por turno e naqueles com carga horária maior.

No que diz respeito aos níveis de burnout nas três dimensões para os médicos, destaca-se a mais alta despersonalização dentre as demais categorias profissionais $(22,5 \%)$. Contudo, houve problemas ao se trabalhar com médias, pelo fato de a amostra ser muito pequena e as idades dos dois médicos investigados serem muito díspares (médico $1=35$ anos e médico $2=70$ anos). O médico mais novo tem jornada tripla de trabalho, com carga horária semanal de 90 horas, em contraponto às 48 horas/semanais do médico mais idoso. Ambos apresentaram mesmo nível de despersonalização, no entanto, os níveis de exaustão emocional (médico $1=40$ e médico $2=9$ ) e de realização profissional (médico $1=37$ e médico 2 =55) evidenciam que o fator idade é essencial na análise dos dados. Sobre a despersonalização, muitos fatores estressores podem contribuir para a diferença como a especialidade médica, a jornada excessiva de 
trabalho, características pessoais como medo de falhar ou não admissão de erros. Contudo, essas são só possibilidades. Pesquisas sobre satisfação no trabalho e o uso de outras escalas de estresse no trabalho, além de pesquisas qualitativas poderiam identificar os agentes estressores que interferem na despersonalização dessa categoria de profissionais.

Os níveis médios de realização profissional identificado em todas as categorias, com exceção ao médico 2, quem elevou a média da categoria médico, podem estar atrelados aos fatores organizacionais já apresentados e discutidos. Uma forma de prevenir a SB e aumentar os níveis de dessa dimensão (realização profissional) seria a compreensão por parte dos profissionais da área da saúde sobre algumas recompensas psicológicas inerentes à área: aliviar a dor e sofrimento de seus pacientes, curar doenças, salvar vidas, diagnosticar corretamente, sentir-se competente, ensinar, advertir, educar, prevenir doenças, receber reconhecimento e gratidão ${ }^{7}$.

\section{CONCLUSÃO}

O presente estudo teve como objetivo identificar o nível de estresse ocupacional, caracterizado como Síndrome de Burnout, em profissionais da área da saúde de um hospital do município de Araçatuba (SP).

O resultado foi obtido por meio da autoaplicação do Maslach Burnout Inventory Human Service Survey - MBI/HSS. A partir deste instrumento, identificaram-se altos níveis de burnout nas dimensões Despersonalização e Exaustão Emocional de auxiliares de Enfermagem, técnicos de Enfermagem, auxiliares de Farmácia e enfermeiros. O nível de Realização Profissional foi médio para todas essas categorias profissionais. Dessa forma, pode-se afirmar que o nível de estresse ocupacional e de Síndrome de Burnout entre esses profissionais é alto.

Já entre os médicos, encontraram-se níveis alto de Despersonalização, médio de Exaustão Emocional e alto de Realização Profissional. Assim, não há a prevalência de SB entre os médicos investigados.

Entende-se que a área da saúde e o ambiente hospitalar, igualmente, sofrem com os fatores organizacionais presentes nos ambientes organizacionais de outras profissões, os quais favorecem o desenvolvimento do estresse ocupacional. Instabilidade, insegurança no trabalho, condições precárias de trabalho, longas jornadas, conflitos nas relações interpessoais com colegas, alta demanda de serviços pela população em geral, crise do sistema de saúde em muitas localidades, entre outros, são fatores que alertam para a necessidade de um olhar mais cuidadoso para a saúde mental desses trabalhadores cujo objetivo maior é o cuidado com o outro.
Diante dos elevados níveis das dimensões da síndrome, entende-se a necessidade de o setor de saúde rever suas políticas e práticas de gestão. Entre as práticas de gestão poderiam estar aquelas que envolvem os programas de qualidade de vida no trabalho e educação contínua no provimento de melhores condições de trabalho ${ }^{7}$. Diagnóstico, implantação de melhorias e inovações gerenciais, tecnológicas e estruturais, que visassem a propiciar condições plenas de desenvolvimento humano durante o trabalho ${ }^{24}$ e que melhorassem as condições de segurança e saúde no trabalho, pois este não deve ocupar o espaço total na vida do trabalhador em detrimento de sua vida particular e familiar.

O estresse ocupacional retrata uma determinada realidade social e histórica de uma profissão. Assim, futuros estudos poderiam continuar se preocupando com amostras multifuncionais maiores, e com pesquisas qualitativas naquelas que apresentarem diferenças expressivas nos níveis de burnout. Também, estudos epidemiológicos poderiam identificar os agentes estressores que qualificam o estresse ocupacional e a SB. Por fim, estudos que façam relações entre esses fatores e a estrutura organizacional nas quais se inserem esses profissionais.

\section{REFERÊNCIAS}

1. Limongi-França AC, Rodrigues AL. Stresse e Trabalho: Uma Abordagem Psicossomática. São Paulo: Atlas; 2012.

2. Fernandes AV. Os antecedentes da Síndrome de Burnout em comerciais de um banco de retalho e os seus efeitos sobre o comprometimento afetivo [dissertação]. Porto: Faculdade de Economia, Universidade do Porto; 2012.

3. Lipp MEN. Pesquisas sobre stress no Brasil: saúde, ocupações e grupos de risco. Campinas: Papirus; 2001.

4. Zanatta AB, Lucca SR. Prevalence of Burnout syndrome in health professionals of an oncohematological pediatric hospital. Rev Esc Enferm. USP. 2015;49(2):251-8.

5. Maslach C. Burnout: A multidimensionalidade perspective. In: Shaufeli, WB, Maslach, C, Marek T. (ed.), Professional burnout: Recet developments in theory and research. Washington, DC: Taylor \& Francis;1993;pp19-32.

6. Maslach C, Leiter MP. Trabalho: fonte de prazer ou desgaste? Guia para vencer o estresse na empresa. Campinas: Papirus; 1999.

7. Carlotto MS, Câmara SG. Propriedades psicométricas do Maslach Burnout Inventory em uma amostra multifuncional. Estud Psicol. 2007;24(3):325-32. 
8. Guido LA, Silva RM, Goulart CT, Bolzan MEO, Lopes LFD. Síndrome de Burnout em residentes multiprofissionais de uma universidade pública. Rev. Esc. Enferm. USP. 2012;46(6):1477-83.

9. Trigo TR. Validade fatorial do Maslach Burnout Inventory-Human Service Survey (MBI-HSS) em uma amostra Brasileira de auxiliares de Enfermagem de um hospital universitário: influência da depressão [dissertação]. São Paulo: Universidade de São Paulo, 2010.

10. Stacciarini JMR, Tróccoli BT. O estresse na atividade ocupacional do enfermeiro. Rev LatinoAm Enfermagem. 2001;9(2):17-25.

11. Schmidt DRC; Dantas RAS, Marziale MHP, Laus AM. Estresse Ocupacional entre Profissionais de Enfermagem do Bloco Cirúrgico. Texto Contexto Enferm. 2009;18(2):330-7.

12. Benevides-Pereira AMT. O Estado da Arte do Burnout no Brasil. Rev Elet InterAção Psy. 2003;1(1):4-11.

13. Creswell JW. Projeto de pesquisa: métodos qualitativo, quantitativo e misto. Porto Alegre: Artmed; 2007.

14. Benevides-Pereira AMT. MBI - Maslach Burnout Inventory e suas adaptações para o Brasil. In: Anais da XXXII Reunião Anual de Psicologia. Rio de Janeiro. 2001:84-85.

15. Codo W. Educação: carinho e trabalho. Petrópolis: Vozes; 1999.

16. Nicoletti IG. Estudo de Evidência de Validade para o Maslach Burnout Inventory (MBI HSS) [dissertação]. Itatiba: Universidade São Francisco; 2011.

17. Noro NTT. Síndrome de Burnout entre Trabalhadores de um Hospital Geral. 2004. [dissertação]. Porto Alegre: Universidade Federal do Rio Grande do Sul; 2004.

18. Vicente CS, Oliveira RA, Maroco J. Factorial validity of the Maslach Burnout Inventory (MBIHSS) among Portuguese professionals. Psic., Saúde \& Doenças. 2013;14(1):152:67.

19. Murofuse NT, Abranches SS, Napoleão AA. Reflexões sobre estresse e Burnout e a relação com a Enfermagem. Rev. Latino-Am Enfermagem. 2005;13(2):255-61.

20. Fernandes SMBA, Medeiros SM, Ribeiro LM. Estresse ocupacional e o mundo do trabalho atual: repercussões na vida cotidiana de enfermeiras. Rev Elet Enferm. 2008;10(2):414-27.

21. Castanheira FVS. Burnout no serviço ao cliente: O Papel das Organizações na Criação de Ambientes de Trabalho Saudáveis [tese]. Lisboa: Universidade de Lisboa; 2009.

22. Roque JD. Burnout e Percepção do Absentismo em Trabalhadores do Setor Bancário [dissertação]. Lisboa: Universidade de Lisboa; 2008.
23. Gomes AR, Cruz JF, Cabanelas S. Estresse ocupacional em profissionais de saúde: um estudo com enfermeiros portugueses. Psic Teor e Pesq. 2009;25(3):307-18.

24. Bitencourt C. Gestão Contemporânea de Pessoas. Novas práticas, Conceitos Tradicionais. Porto Alegre: Artmed; 2004.

\section{CONFLITO DE INTERESSES}

Os autores declaram não haver conflitos de interesse.

\section{AUTOR PARA CORRESPONDÊNCIA}

\section{Diogo Reatto}

orium21@hotmail.com 